Modules over group rings of locally soluble groups with a certain condition of minimality

\author{
O. YU. Dashkova
}




\title{
MODULES OVER GROUP RINGS OF LOCALLY SOLUBLE GROUPS WITH A CERTAIN CONDITION OF MINIMALITY
}

\author{
O. YU. DASHKOVA
}

Received 10 May, 2014

\begin{abstract}
Let $A$ be an $\mathbf{R} G$-module, where $\mathbf{R}$ is an associative ring, $A / C_{A}(G)$ is an infinite $\mathbf{R}$ module, $C_{G}(A)=1, G$ is a locally soluble group. Let $L_{n f}(G)$ be the system of all subgroups $H \leq G$ such that quotient modules $A / C_{A}(H)$ are infinite R-modules. The author studies an $\mathbf{R} G$-module $A$ such that $L_{n f}(G)$ satisfies the minimal condition as an ordered set. It is proved that a locally soluble group $G$ with these conditions is soluble. The structure of $G$ is described.
\end{abstract}

2010 Mathematics Subject Classification: 20F19; 20H25

Keywords: group ring, locally soluble group, module

\section{INTRODUCTION}

Let $A$ be a vector space over a field $F$. The subgroups of the group $G L(F, A)$ of all automorphisms of $A$ are called linear groups. If $A$ has a finite dimension over $F$ then $G L(F, A)$ can be considered as the group of non-singular $(n \times n)$-matrices, where $n=\operatorname{dim}_{F} A$. Finite dimensional linear groups have played an important role in various fields of mathematics, physics and natural sciences, and have been studied many times. When $A$ is infinite dimensional over $F$, the situation is totally different. Infinite dimensional linear groups have been investigated little. The study of this class of groups requires additional restrictions. In [5] it was introduced the definition of the central dimension of an infinite dimensional linear group. Let $H$ be a subgroup of $G L(F, A) . H$ acts on the quotient space $A / C_{A}(H)$ in a natural way. The authors define centdim $F$ to be $\operatorname{dim}_{F}\left(A / C_{A}(H)\right)$. The subgroup $H$ is said to have a finite central dimension if centdim $F H$ is finite and $H$ has infinite central dimension otherwise. Let $G \leq G L(F, A)$. In [5] it was considered the system $L_{i d}(G)$ of all subgroups of $G$ of infinite central dimension. In order to investigate infinite dimensional linear groups that are close to finite dimensional, it is natural to consider the case where the system $L_{i d}(G)$ is "very small". The authors have studied locally soluble infinite dimensional linear groups such that $L_{i d}(G)$ satisfies the minimal condition as an ordered set [5]. 
If $G \leq G L(F, A)$ then $A$ can be considered as an $F G$-module. The natural generalization of this case is the consideration of an $\mathbf{R G}$-module $A$, where $\mathbf{R}$ is a ring whose structure is near to a field. At this point the generalization of the notion of the central dimension of a subgroup of a linear group is the notion of the cocentralizer of a subgroup. This notion was introduced in [8]. Let $A$ be an $\mathbf{R} G$-module, $\mathbf{R}$ be an associative ring, $G$ be a group. If $H \leq G$ then the quotient module $A / C_{A}(H)$ considered as an $\mathbf{R}$-module is called the cocentralizer of $H$ in the module $A$.

Modules over group rings of finite groups have been considered by many authors. Recently this class of modules was investigated in [6]. Study of modules over group rings of infinite groups requires some additional restrictions as in the case of infinite dimensional linear groups. In [2] it was investigated an $\mathbf{R} G$-module $A$ such that $\mathbf{R}$ is a dedekind domain and the cocentralizer of $G$ in the module $A$ is not an artinian $\mathbf{R}$-module. It was considered the system $L_{n a d}(G)$ of all subgroups of $G$ such that their cocentralizers in the module $A$ are not artinian $\mathbf{R}$-modules which is ordered by the usual inclusion. It is investigated an $\mathbf{R} G$-module $A$ such that the system $L_{\text {nad }}(G)$ satisfies the minimal condition as an ordered set, $G$ is a locally soluble group, $C_{G}(A)=1$. The analogous problem for the ring of integers $\mathbf{R}$ was investigated in [3].

In [1] we have studied an $\mathbf{R} G$-module $A$ such that $\mathbf{R}$ is the ring of integers, the cocentralizer of $G$ in the module $A$ is not a noetherian R-module and $C_{G}(A)=1$. Let $L_{n n d}(G)$ be the system of all subgroups of $G$ such that their cocentralizers in the module $A$ are not noetherian $\mathbf{R}$-modules. It was investigated an $\mathbf{R} G$-module $A$ such that $L_{n n d}(G)$ satisfies the minimal condition as an ordered set and $G$ is locally soluble.

In [4] we have considered the similar problem where $\mathbf{R}$ is the ring of integers and the noetherian condition is replaced by the minimax condition.

In this paper we investigate $\mathbf{R} G$-module, where $\mathbf{R}$ is an associative ring, $A / C_{A}(G)$ is an infinite $\mathbf{R}$-module, $C_{G}(A)=1, G$ is a locally soluble group. Let $L_{n f}(G)$ be the system of all subgroups $H \leq G$ such that $A / C_{A}(H)$ are infinite $\mathbf{R}$-modules. We study an $\mathbf{R} G$-module $A$ such that $L_{n f}(G)$ satisfies the minimal condition as an ordered set. It is proved that a locally soluble group $G$ with these conditions is soluble and the structure of $G$ is described.

The main results of this paper are Theorems 2 and 3.

\section{PRELIMINARY RESULTS}

We reduce some elementary facts about $\mathbf{R} G$-modules.

Later on it is considered an $\mathbf{R} G$-module $A$ such that $C_{G}(A)=1$.

Let $A$ be an $\mathbf{R} G$-module where $G$ is a group, $\mathbf{R}$ is an associative ring. Recall that if $K \leq H \leq G$ and the cocentralizer of $H$ in the module $A$ is a finite $\mathbf{R}$-module then the cocentralizer of $K$ in the module $A$ is a finite $\mathbf{R}$-module also. If $U, V$ are 
subgroups of $G$ such that their cocentralizers in the module $A$ are finite $\mathbf{R}$-modules, then $A /\left(C_{A}(U) \cap C_{A}(V)\right)$ is a finite $\mathbf{R}$-module also.

Suppose that a group $G$ satisfies the condition $\min -n f$. If $H_{1}>H_{2}>H_{3}>\cdots$ is an infinite strictly descending chain of subgroups of $G$ then there is the natural number $n$ such that the cocentralizer of $H_{n}$ in the module $A$ is a finite $\mathbf{R}$-module. Moreover, if $N$ is a normal subgroup of $G$ and the cocentralizer of $N$ in the module $A$ is an infinite $\mathbf{R}$-module then $G / N$ satisfies the minimal condition on subgroups.

Lemma 1. Let $A$ be an $\mathbf{R} G$-module, $G$ be a group, $\mathbf{R}$ be an associative ring. Suppose that $G$ satisfies the condition min $-n f, X, H$ are subgroups of $G$ and $\Lambda$ is an index set such that

(1) $X=D r_{\lambda \in \Lambda} X_{\lambda}$, where $1 \neq X_{\lambda}$ is an $H$-invariant subgroup of $X$, for each $\lambda \in \Lambda$.

(2) $H \cap X \leq D r_{\lambda \in \Gamma} X_{\lambda}$ for some subset $\Gamma$ of $\Lambda$.

If the set $\Omega=\Lambda \backslash \Gamma$ is infinite, then the cocentralizer of $H$ in the module $A$ is a finite $\mathbf{R}$-module.

Proof. Suppose that the set $\Omega$ is infinite and let $\Omega_{1} \supset \Omega_{2} \supset \cdots$ be a strictly descending chain of subsets of the set $\Omega$. Since $H \cap D r_{\lambda \in \Omega} X_{\lambda}=1$, the chain of subgroups $\left\langle H, X_{\lambda} \mid \lambda \in \Omega_{1}\right\rangle>\left\langle H, X_{\lambda} \mid \lambda \in \Omega_{2}\right\rangle>\cdots$ is strictly descending. It follows that for some natural number $d$ the cocentralizer of the subgroup $\left\langle H, X_{\lambda} \mid \lambda \in \Omega_{d}\right\rangle$ in the module $A$ is a finite $\mathbf{R}$-module. Therefore the cocentralizer of $H$ in the module $A$ is a finite $\mathbf{R}$-module also.

Lemma 2. Let $A$ be an $\mathbf{R} G$-module, $G$ be a group, $\mathbf{R}$ be an associative ring, $G$ satisfy the condition min-nf, $H, K$ be subgroups of $G$ such that $K$ is a normal subgroup of $H$. Suppose that there exists an index set $\Lambda$ and subgroups $H_{\lambda}$ of $G$ such that $K \leq H_{\lambda}$ for all $\lambda \in \Lambda, H / K=D r_{\lambda \in \Lambda} H_{\lambda} / K$, and the set $\Lambda$ is infinite. Then the cocentralizer of $H$ in the module $A$ is a finite $\mathbf{R}$-module.

Proof. Let $\Gamma$ and $\Omega$ are infinite disjoint subsets of the set $\Lambda$ such that $\Lambda=\Gamma \cup \Omega$. Let $U / K=D r_{\lambda \in \Gamma} H_{\lambda} / K$, let $V / K=D r_{\lambda \in \Omega} H_{\lambda} / K$, and let $\Gamma_{1} \supset \Gamma_{2} \supset \cdots$ be a strictly descending chain of subsets of the set $\Gamma$. Then we construct an infinite strictly descending chain of subgroups

$$
\left\langle V, H_{\lambda} \mid \lambda \in \Gamma_{1}\right\rangle>\left\langle V, H_{\lambda} \mid \lambda \in \Gamma_{2}\right\rangle>\cdots .
$$

It follows from the condition min- $n f$ that the cocentralizer of $V$ in the module $A$ is a finite $\mathbf{R}$-module. Likewise, we obtain that the cocentralizer of $U$ in the module $A$ is a finite $\mathbf{R}$-module. Since $H=U V$, it follows that the cocentralizer of $H$ in the module $A$ is a finite $\mathbf{R}$-module also.

Lemma 3. Let $A$ be an $\mathbf{R} G$-module, $G$ be a group, $\mathbf{R}$ be an associative ring, $G$ satisfy the condition min $-n f$. If an element $g \in G$ has infinite order then the cocentralizer of $\langle g\rangle$ in the module $A$ is a finite $\mathbf{R}$-module. 
Proof. Let $p, q$ are distinct primes greater than 3 and let $u=g^{p}, v=g^{q}$. Then there is an infinite descending chain of subgroups $\langle u\rangle>\left\langle u^{2}\right\rangle>\left\langle u^{4}\right\rangle>\ldots$. It follows from the condition min-nf that there exists the natural number $k$ such that the cocentralizer of the subgroup $\left\langle u^{2^{k}}\right\rangle$ in the module $A$ is a finite $\mathbf{R}$-module. Similarly, there exists a natural number $l$ such that the cocentralizer of the subgroup $\left\langle v^{3^{l}}\right\rangle$ in the module $A$ is a finite $\mathbf{R}$-module. Therefore the cocentralizer of the subgroup $\langle g\rangle=\left\langle u^{2^{k}}\right\rangle\left\langle v^{3^{l}}\right\rangle$ in the module $A$ is a finite $\mathbf{R}$-module.

The following result gives an important information about the derived quotient group under the condition $\min -n f$.

Lemma 4. Let $A$ be an $\mathbf{R} G$-module, $G$ be a group, $\mathbf{R}$ be an associative ring. Suppose that the cocentralizer of $G$ in the module $A$ is an infinite $\mathbf{R}$-module, and $G$ satisfies the condition min $-n f$. Then the quotient group $G / G^{\prime}$ is Chernikov.

Proof. Suppose that the quotient group $G / G^{\prime}$ is not Chernikov group. Let $\mathfrak{S}$ be the family of all subgroups $H \leq G$ such that the quotient group $H / H^{\prime}$ is not Chernikov and the cocentralizer of $H$ in the module $A$ is an infinite $\mathbf{R}$-module. Since $G \in \mathfrak{S}$ then $\mathfrak{S} \neq \varnothing$. Since the set $\mathfrak{S}$ satisfies the minimal condition, then it has a minimal element. Let $D$ be this minimal element. If $U, V$ are proper subgroups of the group $D$ such that $D=U V$ and $U \cap V=D^{\prime}$, then at least one of these subgroups, $U$ say, such that its cocentralizer in the module $A$ is an infinite $\mathbf{R}$-module. The choice of $D$ implies that $U / U^{\prime}$ is Chernikov. It follows with regard to the isomorphism $U / D^{\prime} \simeq\left(U / U^{\prime}\right) /\left(D^{\prime} / U^{\prime}\right)$ that $U / D^{\prime}$ is also Chernikov. Since the cocentralizer of $U$ in the module $A$ is an infinite $\mathbf{R}$-module it follows that the abelian quotient group $D / U$ is also Chernikov. Hence the quotient group $D / D^{\prime}$ is Chernikov. Contrary to the choice of $D$. Therefore $D / D^{\prime}$ is indecomposable. Hence $D / D^{\prime}$ is isomorphic to a subgroup of quasi-cyclic group $C_{q} \infty$, for some prime $q$. Contradiction.

Let $A$ be an $\mathbf{R} G$-module, $G$ be a group, $\mathbf{R}$ be an associative ring. Let $F F D(G)$ be the set of all elements $x \in G$ such that the cocentralizer of $\langle x\rangle$ in the module $A$ is a finite $\mathbf{R}$-module. Since $C_{A}\left(x^{g}\right)=C_{A}(x) g$ for all $x, g \in G$, it follows that $F F D(G)$ is a normal subgroup of $G$.

Lemma 5. Let $A$ be an $\mathbf{R} G$-module, $G$ be a group, $\mathbf{R}$ be an associative ring. Suppose that the cocentralizer of $G$ in the module $A$ is an infinite $\mathbf{R}$-module, and $G$ satisfies the condition min $-n f$. Then $G$ is either periodic or $G=F F D(G)$.

Proof. We suppose to the contrary that $G$ is neither periodic nor $G \neq F F D(G)$. Let $\mathfrak{S}$ be the family of all subgroups $H \leq G$ such that $H$ is not periodic and $H \neq$ $F F D(H)$. $\mathfrak{S}$ is non-empty. If $H \neq F F D(H)$ then there is an element $h \in H$ such that the quotient module $A / C_{A}(h)$ is an infinite $\mathbf{R}$-module. Hence $\mathfrak{S} \subseteq L_{n f}(G)$, and 
therefore $\mathfrak{S}$ satisfies the minimal condition. Let $D$ be the minimal element of $\mathfrak{S}$, let $L=F F D(D)$. Note that $L \neq 1$, since $D$ is not a periodic group. If $L \leq S \leq D$ and $S \neq D$, then $S=F F D(S)$ so $S \leq L$. Hence $D / L$ has order $q$ for some prime $q$. Let $x \in D \backslash L$. If an element $a$ has infinite order, then the choice of $D$ implies that $\langle x, a\rangle=D$. It follows that $L$ is finitely generated and since $L=F F D(L)$, the quotient module $A / C_{A}(L)$ is a finite $\mathbf{R}$-module. Since the subgroup $L$ is normal in $D$, then $C=C_{A}(L)$ is an $\mathbf{R} D$-submodule of $A$. It follows that $A$ has the finite series of $\mathbf{R} D$-submodules

$$
\langle 0\rangle \leq C \leq A,
$$

such that $A / C$ is a finite $\mathbf{R}$-module. Since $A / C$ is a finite $\mathbf{R}$-module then $D / C_{D}(A / C)$ is finite. As $C=C_{A}(L)$ then $L \leq C_{D}(C)$. It follows that $D / C_{D}(C)$ is finite too.

Let $W=C_{D}(C) \cap C_{D}(A / C)$. By Remak theorem

$$
D / W \leq D / C_{D}(C) \times D / C_{D}(A / C) .
$$

It follows that the quotient group $D / W$ is finite. $W$ acts trivially on each factor of the series $\langle 0\rangle \leq C \leq A$. Therefore $W$ is abelian.

Let $U$ be a normal subgroup of finite index of $D$. The subgroup $U$ is not periodic and so $\langle U, x\rangle$ is neither periodic nor $\langle U, x\rangle \neq F F D(\langle U, x\rangle)$. The choice of $D$ implies that $D=\langle U, x\rangle$ and hence the quotient group $D / U$ is abelian. If $E$ is the finite residual of $D$, it follows that the quotient group $D / E$ is abelian. Since $E \leq W$ then $D / W$ is also abelian. It follows that $D /(W \cap L)$ is abelian. Since $W \cap L \leq W$, then the subgroup $W \cap L$ is abelian, and so $D$ is a finitely generated metabelian subgroup. By theorem of P.Hall (Theorem 9.51 [9]) $D$ is residually finite. As above, $D$ is therefore abelian. Since $D=U\langle x\rangle$ for every subgroup $U$ of finite index, it follows that the group $D$ is infinite cyclic. By Lemma $3 D=F F D(D)$. We have the contradiction with the choice of $D$.

\section{LOCALLY SOLUBLE GROUPS WITH THE CONDITION $\min -n f$}

Lemma 6. Let $A$ be an $\mathbf{R} G$-module, $G$ be a periodic locally soluble group, $\mathbf{R}$ be an associative ring. Suppose that the cocentralizer of $G$ in the module $A$ is an infinite $\mathbf{R}$-module and $G$ satisfies the condition min $-n f$. Then $G$ either satisfies the minimal condition on subgroups or $G=F F D(G)$.

Proof. We suppose to the contrary that $G$ is neither satisfies the minimal condition on subgroups nor $G \neq F F D(G)$. Let $\mathfrak{S}$ be the family of all subgroups $H \leq G$ such that $H$ does not satisfy the minimal condition on subgroups and $H \neq F F D(H)$. Then $\mathfrak{S} \neq \varnothing$. If $H \neq F F D(H)$ then the cocentralizer of $H$ in the module $A$ is an infinite R-module and hence $\mathfrak{S} \subseteq L_{n f}(G)$. Therefore $\mathfrak{S}$ satisfies the minimal condition. Let $D$ be the minimal element and let $L=F F D(D)$. There exists an infinite strictly descending chain of subgroups of $D$ : 


$$
H_{1}>H_{2}>H_{3}>\cdots .
$$

Since $D$ satisfies the condition $\min -n f$ then there exists the natural number $k$ such that the cocentralizer of $H_{k}$ in the module $A$ is a finite $\mathbf{R}$-module. Therefore $H_{k} \leq L$, and hence $L$ does not satisfy the minimal condition. If $x \in D \backslash L$ then it follows from the choice of the subgroup $D$ that $\langle x, L\rangle=D$. Hence the quotient group $D / L$ has the order $q$ for prime $q$. If it is necessary we replace $x$ by the suitable power and obtain that $x$ has the order $q^{r}$ for some natural number $r$. Since the group $D$ is not Chernikov then by D.I.Zaicev's theorem [10], $D$ contains $\langle x\rangle$-invariant abelian subgroup $B=$ $D r_{n \in \mathbf{N}}\left\langle b_{n}\right\rangle$ and we may assume that the elements $b_{n}$ have prime orders for all $n \in$ $\mathbf{N}$. Let $1 \neq c_{1} \in B$ and $C_{1}=\left\langle c_{1}\right\rangle^{\langle x\rangle}$. Then $C_{1}$ is finite and there is the subgroup $E_{1}$ such that $B=C_{1} \times E_{1}$. Let $U_{1}=\operatorname{core}_{\langle x\rangle} E_{1}$. Therefore $U_{1}$ has finite index in $B$. If $1 \neq c_{2} \in U_{1}$ and $C_{2}=\left\langle c_{2}\right\rangle^{\langle x\rangle}$ then $C_{2}$ is a finite $\langle x\rangle$-invariant subgroup and $\left\langle C_{1}, C_{2}\right\rangle=C_{1} \times C_{2}$. Continuing in this manner, we can construct a family of subgroups $\left\{C_{n} \mid n \in \mathbf{N}\right\}$ for which $\left\langle C_{n} \mid n \in \mathbf{N}\right\rangle=D r_{n \in \mathbf{N}} C_{n}$. By Lemma $1 x \in L$. Contradiction.

From Lemmas 5 and 6 it follows the theorem.

Theorem 1. Let $A$ be an $\mathbf{R} G$-module, $G$ be a locally soluble group, $\mathbf{R}$ be an associative ring. Suppose that the cocentralizer of $G$ in the module $A$ is an infinite $\mathbf{R}$ module, and $G$ satisfies the condition min $-n f$. Then $G$ either satisfies the minimal condition on subgroups or $G=F F D(G)$.

Lemma 7. Let $A$ be an $\mathbf{R} G$-module, $G$ be a locally soluble group. Suppose that the cocentralizer of $G$ in the module $A$ is a finite $\mathbf{R}$-module. Then $G$ is almost abelian.

Proof. Let $C=C_{A}(G)$. Then $A$ has the series of $\mathbf{R} G$-submodules $\langle 0\rangle \leq C \leq A$, where $A / C$ is a finite $\mathbf{R}$-module. Since $G \leq C_{G}(C)$ then $G / C_{G}(C)$ is trivial. As $A / C$ is a finite $\mathbf{R}$-module then $G / C_{G}(A / C)$ is finite.

Let $H=C_{G}(C) \cap C_{G}(A / C)$. Each element of $H$ acts trivially on every factor of the series $\langle 0\rangle \leq C \leq A / C$. By Kaluzhnin Theorem (p. 144 [7]) $H$ is abelian. By Remak's Theorem

$$
G / H \leq G / C_{G}(C) \times G / C_{G}(A / C) .
$$

It follows that $G / H$ is finite. Then $G$ is an almost abelian group.

Lemma 8. Let $A$ be an $\mathbf{R} G$-module, $G$ be a locally soluble group, $\mathbf{R}$ be an associative ring, and if the cocentralizer of $G$ in the module $A$ is an infinite $\mathbf{R}$-module then $G$ satisfies the condition min $-n f$. Then either $G$ is soluble or $G$ has an ascending series of normal subgroups $1=W_{0} \leq W_{1} \leq \cdots \leq W_{n} \leq \cdots \leq W_{\omega}=\cup_{n \in \mathbf{N}} W_{n} \leq G$, 
such that the cocentralizer of each subgroup $W_{n}$ in the module $A$ is a finite $\mathbf{R}$-module, the factors $W_{n+1} / W_{n}$ are abelian for $n=1,2, \cdots$, and $G / W_{\omega}$ is a Chernikov group.

Proof. If the quotient module $A / C_{A}(G)$ is a finite $\mathbf{R}$-module then $G$ is soluble by Lemma 7. Therefore it seemed reasonable to study locally soluble groups $G$ such that $A / C_{A}(G)$ is an infinite $\mathbf{R}$-module.

Later we consider the case when the cocentralizer of $G$ in the module $A$ is an infinite $\mathbf{R}$-module. At first we prove that $G$ is hyperabelian. To accomplish this we show that every non-trivial image of $G$ contains a non-trivial normal abelian subgroup.

Let $H$ be a proper normal subgroup of $G$. Suppose that the cocentralizer of $H$ in the module $A$ is an infinite $\mathbf{R}$-module. Then $G / H$ satisfies the minimal condition on subgroups. Therefore $G / H$ is Chernikov group, and contains a non-trivial normal abelian subgroup. Now we suppose that the the cocentralizer of $H$ in the module $A$ is a finite $\mathbf{R}$-module. Let $\mathfrak{L}=\left\{M_{\sigma} / H \mid \sigma \in \Sigma\right\}$ be the family of all non-trivial normal subgroups of the quotient group $G / H$. At first we consider the case when for all $\sigma \in$ $\Sigma$ the cocentralizer of $M_{\sigma}$ in the module $A$ is an infinite $\mathbf{R}$-module. We shall prove that the quotient group $G / H$ satisfies the minimal condition on normal subgroups. Let $\left\{M_{\delta} / H\right\}$ be a non-empty subset of $\mathfrak{L}$. The cocentralizer of a subgroup $M_{\delta}$ in the module $A$ is an infinite $\mathbf{R}$-module for all $\delta$. By the condition min-nf the set $\left\{M_{\delta}\right\}$ has the minimal element $M$. Then $M / H$ is the minimal element of subset $\left\{M_{\delta} / H\right\}$. Therefore $G / H$ satisfies the minimal condition on normal subgroups. It follows that the quotient group $G / H$ is hyperabelian and contains a non-trivial normal abelian subgroup. In the case when for some $\gamma \in \Sigma$ the cocentralizer of $M_{\gamma}$ in the module $A$ is a finite $\mathbf{R}$-module, the subgroup $M_{\gamma}$ is soluble. Then $M_{\gamma} / H$ is a non-trivial normal soluble subgroup of $G / H$. Therefore the quotient group $G / H$ contains a non-trivial normal abelian subgroup and so $G$ is hyperabelian.

Let $1=H_{0} \leq H_{1} \leq \cdots \leq H_{\alpha} \leq \cdots \leq G$ be a normal ascending series with abelian factors and let $\alpha$ be the least ordinal such that the cocentralizer of $H_{\alpha}$ in the module $A$ is an infinite $\mathbf{R}$-module. Then, as above, the subgroup $H_{\beta}$ is soluble for all $\beta<\alpha$. Moreover, the quotient group $G / H_{\alpha}$ satisfies the minimal condition on subgroups, and so is a soluble Chernikov group.

At first we suppose that $\alpha$ is not a limit ordinal. Then the subgroup $H_{\alpha}$ is soluble and it follows that $G$ is soluble also. Now we consider the case when $\alpha$ is a limit ordinal, and $G$ is not soluble. For all natural numbers $k$ there exists an ordinal $\beta_{k}$ such that $\beta_{k}<\alpha, H_{\beta_{k}}$ has derived length at least $k$. Moreover, we may assume that $\beta_{i}<\beta_{i+1}$ for all natural numbers $i$. Let $T_{i}=H_{\beta_{i}}$ for all natural numbers $i$. It follows that $G$ has an ascending series of normal soluble subgroups $1=T_{0} \leq T_{1} \leq$ $\cdots \leq \cdots$. Then the subgroup $T_{\omega}=\cup_{n \in \mathbf{N}} T_{n}$ is not soluble and so $T_{\omega}=H_{\alpha}$. A series $1=W_{0} \leq W_{1} \leq \cdots \leq W_{n} \leq \cdots \leq W_{\omega}=\cup_{n \in \mathrm{N}} W_{n} \leq G$ with the propeties referred in the theorem can be obtained from the series $1=T_{0} \leq T_{1} \leq \cdots \leq T_{\omega} \leq G$. 
Lemma 9. Let $A$ be an $\mathbf{R} G$-module, $G$ be a group, $\mathbf{R}$ be an associative ring. Suppose that the cocentralizer of $G$ in the module $A$ is an infinite $\mathbf{R}$-module, $G$ satisfies the condition min $-n f$ and $G=F F D(G)$. Then the quotient group $G / G^{\Im}$ is finite.

Proof. We suppose for a contradiction that the quotient group $G / G^{\Im}$ is infinite. Then $G$ has an infinite strictly descending series of normal subgroups $G>N_{1}>$ $N_{2}>\cdots$, such that the quotient groups $G / N_{i}$ are finite for each $i$. Therefore there exists $k$ for which the quotient group $G / N_{k}$ is finite and the cocentralizer of $N_{k}$ in the module $A$ is a finite $\mathbf{R}$-module. Since $G=F F D(G)$, there is the subgroup $H$ such that its cocentralizer in the module $A$ is a finite $\mathbf{R}$-module and $G=H N_{k}$. Hence the cocentralizer of $G$ in the module $A$ is a finite $\mathbf{R}$-module. Contradiction.

Lemma 10. Let $A$ be an $\mathbf{R} G$-module, $G$ be a locally soluble group, $\mathbf{R}$ be an associative ring. Suppose that the cocentralizer of $G$ in the module $A$ is an infinite $\mathbf{R}$-module and $G$ satisfies the condition min $-n f$. If $G$ has an ascending series of normal subgroups $1=W_{0} \leq W_{1} \leq \cdots \leq W_{n} \leq \cdots \leq \cup_{n \geq 1} W_{n}=G$, in which the cocentralizer of each subgroup $W_{n}$ in the module $A$ is a finite $\mathbf{R}$-module, and each factor $W_{n+1} / W_{n}$ is abelian, then $G$ is soluble.

Proof. Since the quotient module $A / C_{A}\left(W_{k}\right)$ is a finite $\mathbf{R}$-module for each $k \in$ $\mathbb{N}$ then there is the series of $\mathbf{R} G$-submodules $A=A_{0} \geq A_{1} \geq A_{2} \geq \cdots \geq A_{k} \geq$ $\cdots \geq A_{\omega}=C_{A}(G)$, such that $A_{k}=C_{A}\left(W_{k}\right)$ and each factor $A_{k} / A_{k+1}$ is a finite $\mathbf{R} G$-module. Let $H=\cap_{j \geq 0} C_{G}\left(A_{j} / A_{j+1}\right)$. Then $G / C_{G}\left(A_{j} / A_{j+1}\right)$ is finite for each $j \in \mathbb{N}$. Since $G / H$ embeds in the Cartesian product of the quotient groups $G / C_{G}\left(A_{j} / A_{j+1}\right)$, it follows that $G / H$ is residually finite. Moreover, $G$ is a union of subgroups such that their cocentralizers in the module $A$ are finite $\mathbf{R}$-modules. Hence $G=F F D(G)$. By Lemma 9 the quotient group $G / H$ is finite.

Since $G=F F D(G)$ then the cocentralizer of $H$ in the module $A$ is an infinite R-module. We shall prove that $H$ is soluble. Let $L_{j}=C_{H}\left(A / A_{j}\right), j=1,2, \cdots$. Let $H \neq L_{j}$ for some $j$. The quotient group $H / L_{j}$ is finite for each $j=1,2, \cdots$. We suppose that there is the number $j$ such that the cocentralizer of $L_{j}$ in the module $A$ is a finite $\mathbf{R}$-module. Let $j$ be the least number with this property. It follows that the cocentralizer of $L_{j-1}$ in the module $A$ is an infinite $\mathbf{R}$-module. On the other hand since the quotient group $L_{j-1} / L_{j}$ is finite and $G=F F D(G)$, then the cocentralizer of $L_{j-1}$ in the module $A$ is a finite $\mathbf{R}$-module. We have contradiction. Therefore the cocentralizer of each subgroup $L_{j}$ in the module $A$ is an infinite $\mathbf{R}$-module. Since $H$ satisfies the condition min $-n f$ then there exists the number $m$ such that $L_{j}=L_{m}$ for all $j \geq m$. From this fact and from the choice of subgroup $L_{j}$ it follows that the subgroup $L_{m}$ is soluble. Since the quotient group $H / L_{m}$ is finite then $H$ is also soluble. Then $G$ is soluble. 
From the obtained results it follows Theorem 2.

Theorem 2. Let $A$ be an $\mathbf{R} G$-module, $G$ be a locally soluble group, $\mathbf{R}$ be an associative ring. Suppose that if the cocentralizer of $G$ in the module $A$ is an infinite $\mathbf{R}$-module, $G$ satisfies the condition min $-n f$. Then $G$ is soluble.

Theorem 3. Let $A$ be an $\mathbf{R} G$-module, $G$ be a locally soluble group, $\mathbf{R}$ be an associative ring. Suppose that the cocentralizer of $G$ in the module $A$ is an infinite $\mathbf{R}$-module and $G$ satisfies the condition min- $n f$. Then $G$ has the normal abelian subgroup $H$ such that $G / H$ is Chernikov.

Proof. It should be noted that by Theorem 2 the group $G$ is soluble. To accomplish this proof we consider the case when $G$ is not Chernikov.

Let $G=D_{0} \geq D_{1} \geq D_{2} \geq \cdots \geq D_{n}=1$ be the derived series of $G$. There exists the number $m$ such that the cocentralizer of $D_{m}$ in the module $A$ is an infinite $\mathbf{R}$ module but the cocentralizer of $D_{m+1}$ in the module $A$ is a finite $\mathbf{R}$-module. By Lemma 4 the quotient groups $D_{i} / D_{i+1}, i=0,1, \ldots, m$, are Chernikov. Let $U=$ $D_{m+1}$. Then the quotient group $G / U$ is Chernikov. Let $C=C_{A}(U) . C$ is an $\mathbf{R} G$ submodule of $A$. Therefore there exists the series of -submodules

$$
\langle 0\rangle \leq C \leq A,
$$

such that $A / C$ is a finite $\mathbf{R}$-module. Then $G / C_{G}(A / C)$ is finite.

Let $H=C_{G}(C) \cap C_{G}(A / C)$. The subgroup $H$ acts trivially on each factor of the series $\langle 0\rangle \leq C \leq A$. Therefore $H$ is abelian. Since the quotient group $G / U$ is Chernikov and $U \leq C_{G}(C)$ then the quotient group $G / C_{G}(C)$ is also Chernikov. By Remak theorem $G / H \leq G / C_{G}(C) \times G / C_{G}(A / C)$. It follows that $G / H$ is Chernikov. Therefore $G$ contains the normal abelian subgroup $H$ such that $G / H$ is Chernikov.

In the paper the author have used the methods of the proofs of [5].

\section{REFERENCES}

[1] O. Dashkova, "Modules over integer group rings of locally solvable groups with the restrictions on some systems of subgroups. (Russian)," Dopov. Nats. Akad. Nauk Ukr., no. 2, pp. 14-19, 2009.

[2] O. Dashkova, "On a class of modules over group rings of locally soluble groups. (Russian)," Trudy Inst. Mat. i Mech. Ural Otd. Ros. Akad. Nauk, vol. 15, no. 2, pp. 94-98, 2009. [Online]. Available: http://dx.doi.org/10.1134/S0081543809070062

[3] O. Dashkova, "On one class of modules over integer group rings of locally solvable groups. (Russian)," Ukrainian Math. J., vol. 61, no. 1, pp. 44-51, 2009. [Online]. Available: http://dx.doi.org/10.1007/s11253-009-0197-x

[4] O. Dashkova, "Modules over integer group rings of locally soluble groups with minimax restriction. (Russian)," Fundam. Prikl. Mat., vol. 17, no. 3, pp. 25-37, 2011/2012. [Online]. Available: http://dx.doi.org/10.1007/s10958-012-1055-1

[5] M. Dixon, M. Evans, and L. Kurdachenko, "Linear groups with the minimal condition on subgroups of infinite central dimension," J. Algebra, vol. 277, no. 1, pp. 172-186, 2004. [Online]. Available: http://dx.doi.org/10.1016/j.jalgebra.2004.02.029 
[6] P. M. Gudivok, V. P. Rud'ko, and V. A. Bovdi, Crystallographic groups (Ukrainian). Uzhgorod: Uzhgorods'kiı̌ Natsional'niı̌ Universitet, 2006.

[7] M. Kargapolov and Y. Merzlyakov, Fundamentals of group theory. (Russian). Moscow: Izdat. "Nauka", 1972.

[8] L. Kurdachenko, "On groups with minimax classes of conjugate elements," Infinite groups and adjoining algebraic structures, pp. 160-177, 1993.

[9] D. Robinson, Finiteness Conditions and Generalized Soluble Groups, ser. Ergebnisse der Mathematik und ihrer Grenzgebiete. Berlin, Heidelberg, New York: Springer-Verlag, 1972, vol. 1,2.

[10] D. Zaicev, "Solvable subgroups of locally solvable groups," Dokl. Akad. Nauk SSSR, vol. 214, no. 6, pp. 1250-1253, 1974.

Author's address

O. Yu. Dashkova

The Branch of Moscow state university in Sevastopol, Department of Mathematics, 7 Sevastopol heroes street, 99001 Sevastopol, Russia

E-mail address: odashkova@yandex.ru 